\title{
Coupling between reconnection and Kelvin-Helmholtz instabilities in collisionless plasmas
}

\author{
D. Grasso ${ }^{1,2}$, D. Borgogno ${ }^{3}$, F. Pegoraro ${ }^{4}$, and E. Tassi ${ }^{5,2}$ \\ ${ }^{1}$ CNR-INFM, Genova, Italy \\ ${ }^{2}$ Burning Plasma Reasearch Group, Dip. di Energetica, Politecnico di Torino, CNISM, Italy \\ ${ }^{3}$ Université de Nice-Sophia Antipolis, CNRS, OCA, Nice, France \\ ${ }^{4}$ Phys. Dept. Pisa University, Pisa, CNISM, Italy \\ ${ }^{5}$ Centre de Physique Theorique - UMR 6207, CNRS Luminy, Case 907, 13288 Marseille Cedex 09, France
}

Received: 30 December 2008 - Revised: 23 March 2009 - Accepted: 24 March 2009 - Published: 3 April 2009

\begin{abstract}
In a collisionless plasma, when reconnection instability takes place, strong shear flows may develop. Under appropriate conditions these shear flows become unstable to the Kelvin-Helmholtz instability. Here, we investigate the coupling between these instabilities in the framework of a four-field model. Firstly, we recover the known results in the low $\beta$ limit, $\beta$ being the ratio between the plasma and the magnetic pressure. We concentrate our attention on the dynamical evolution of the current density and vorticity sheets which evolve coupled together according to a laminar or a turbulent regime. A three-dimensional extension in this limit is also discussed. Secondly, we consider finite values of the $\beta$ parameter, allowing for compression of the magnetic and velocity fields along the ignorable direction. We find that the current density and vorticity sheets now evolve separately. The Kelvin-Helmholtz instability involves only the vorticity field, which ends up in a turbulent regime, while the current density maintains a laminar structure.
\end{abstract}

\section{Introduction}

Magnetic reconnection is believed to be a crucial mechanism in order to explain different phenomena in laboratory as well as in astrophysical plasmas. Sawtooth oscillations and solar flares are examples of such phenomena. Although magnetic reconnection is a local process, occurring on small scale, its main feature is a rearrangement of the magnetic field lines topology on a global scale. Typically a magnetic island forms

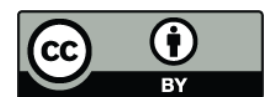

Correspondence to: D. Grasso (daniela.grasso@infm.polito.it) together with vorticity and current density layers. Related to these layers, strong shear flows may develop and, under appropriate conditions, become unstable to a hydrodynamical instability of the Kelvin-Helmholtz type, generating turbulent structures on small scales.

In high temperature plasmas the reconnection time is usually shorter than the electron-ion collision time so that the electron inertia becomes the main mechanism responsible for the reconnection process. Although a kinetic approach should be invoked in order to treat such low collisional regimes, fluid models, which offer a computational advantage, are often used. Thus, a collisionless approach in the framework of the two-dimensional four-field model of Fitzpatrick and Porcelli (2004) is adopted. This model, in the low $\beta$ limit, reduces to the two-field model of Schep et al. (1994). Due to the absence of dissipation, this model admits a Hamiltonian formulation that make it possible to identify the invariants of the system evolution. In the framework of the two-field model it has already been shown that the evolution of the current density and vorticity layers change depending on the value of the electron temperature (Del Sarto et al., 2003, 2005; Grasso et al., 2007). In particular, when the electron temperature is negligible, the strong shear flow that is generated during the reconnection process develops into a turbulent regime under the effect of hydrodynamical instabilities. On the other side, when electron temperature effects are taken into account, the current density and vorticity layers evolve according to a stable laminar structure. This different behavior has been explained in terms of the evolution of the invariants of the system, that undergo a phase mixing process in the presence of finite electron temperature (Grasso et al., 2001). Here we recover these results, adopting the more general four-field model. Incidentally, this difference between

Published by Copernicus Publications on behalf of the European Geosciences Union and the American Geophysical Union. 
cold and hot electron regimes has been also proved in more general 3-D configurations (Grasso et al., 2007). Here we present new simulations of the two-field model in this 3-D context.

When we add more degrees of freedom to the system, allowing for perturbations of the magnetic and velocity fields along the ignorable coordinate, and consider finite $\beta$ regimes, we find that this distinction between laminar and turbulent regimes depending on the value of the electron temperature does not longer apply. Indeed, the Kelvin-Helmholtz type instability may develop on the top of the reconnection process regardless the value of the electron temperature. Indeed, we find that the vorticity layers undergo a Kelvin-Helmholtz instability, while the current density layers evolve according to a laminar structure. This twofold behavior will be explained in terms of the invariants of the model. The paper is organized as follows: in Sect. 2 we introduce the fourfield model; in Sect. 3 its Hamiltonian structure is recalled; in Sect. 4 the low $\beta$ limit of the model is recovered together with its 3-D extension; while in Sect. 5 the new higher $\beta$ regime is presented. Conclusions will end the paper.

\section{Model equations}

The four-field model of Fitzpatrick and Porcelli (2004) is given by the following dimensionless equations:

$$
\begin{array}{r}
\frac{\partial\left(\psi-d_{e}^{2} \nabla^{2} \psi\right)}{\partial t}+\left[\varphi, \psi-d_{e}^{2} \nabla^{2} \psi\right] \\
-d_{\beta}[\psi, Z]=0 \\
\frac{\partial Z}{\partial t}+[\varphi, Z]-c_{\beta}[v, \psi]-d_{\beta}\left[\nabla^{2} \psi, \psi\right]=0, \\
\frac{\partial \nabla^{2} \varphi}{\partial t}+\left[\varphi, \nabla^{2} \varphi\right]+\left[\nabla^{2} \psi, \psi\right]=0, \\
\frac{\partial v}{\partial t}+[\varphi, v]-c_{\beta}[Z, \psi]=0 .
\end{array}
$$

The four fields $\psi, Z, \varphi$ and $v$ are functions of $x, y$ and time $t$, and are related to the magnetic field $\mathbf{B}$ and to the plasma fluid velocity $\mathbf{v}$ by the following relationships: lengths are normalized on the equilibrium variation scale of the magnetic field, $L$, and time is normalized on the Alfvèn time, $\tau_{A}=L / v_{A}$, where the Alfvèn velocity is based on the poloidal magnetic field.

$$
\begin{aligned}
& \mathbf{B}=\left(B_{0}+c_{\beta} Z\right) \mathbf{e}_{z}+\nabla \psi \times \mathbf{e}_{z}, \\
& \mathbf{v}=\mathbf{e}_{z} \times \nabla \varphi+v \mathbf{e}_{z},
\end{aligned}
$$

where $B_{0}$ is a constant guide field, $c_{\beta}=\sqrt{\beta /(1+\beta)}$ with $\beta=(5 / 3) p_{0} / B_{0}$, being $p_{0}$ the constant background plasma pressure, $d_{\beta}=d_{i} c_{\beta}$, while $d_{i}$ and $d_{e}$ indicate the ion and electron skin depth, respectively. The symbol [, ] indicates the canonical Poisson bracket, so that $[f, g]=(\nabla f \times \nabla g) \cdot \hat{\mathbf{z}}$, for generic fields $f$ and $g$. The system of Eqs. (1-4), the derivation of which is based on the assumption that $Z \ll B_{0}$, is obtained from the standard two-fluid description of a plasma. Equation (1) is a reduced Ohm's law where the presence of finite electron inertia, which makes it possible for magnetic reconnection to take place, is indicated by the terms proportional to the electron skin depth $d_{e}$. Equations 2, 3 and 4 are obtained from the electron vorticity equation, the plasma vorticity equation and the parallel plasma momentum equation, respectively.

\section{Hamiltonian structure}

Given the absence of dissipative terms, the set of Eqs. (1-4) is a natural candidate for being a Hamiltonian system. Indeed, the non-canonical Hamiltonian structure of the system has been derived in Tassi et al. (2007) and thoroughly discussed in Tassi et al. (2008b). The derivation of such structure follows from having realized that the functional

$$
H=\frac{1}{2} \int_{\mathcal{D}} d^{2} x\left(d_{e}^{2} J^{2}+|\nabla \psi|^{2}+|\nabla \varphi|^{2}+v^{2}+Z^{2}\right)
$$

is a constant of motion for the system. In Eq. (7) $J=-\nabla^{2} \psi$ is the parallel current density whereas $\mathcal{D}$ is a domain of integration on the boundary of which the fields are supposed to vanish. The functional $H$ represents the total energy of the system, which includes both kinetic and magnetic contributions. The derivation of the Hamiltonian structure of a $n$-field system is completed (see, e.g. Morrison, 1998) when a suitable antisymmetric bilinear form $\{$,$\} (Lie-Poisson bracket),$ satisfying the Jacobi identity is found, such that the model equations can be written in the form

$$
\frac{\partial \xi_{i}}{\partial t}=\left\{\xi_{i}, H\right\}, \quad i=1, \ldots, n,
$$

with $\xi_{i}$ indicating a set of field variables. The expression for the Lie-Poisson bracket of the four-field model in the original physical variables is rather lengthy and can be found in Tassi et al. (2008b). Lie-Poisson brackets are associated to Casimir functionals $C$ (Morrison, 1998), which are constants of motion characterized by the property $\{f, C\}=0$, for every $f$. In the case of the four-field model four infinite families of Casimirs have been found, corresponding to

$$
\begin{aligned}
C_{1} & =\int d^{2} x \omega \mathcal{F}(D), \\
C_{2} & =\int d^{2} x \mathcal{K}(D), \\
C_{ \pm} & =\int d^{2} x g_{ \pm}\left(T_{ \pm}\right) .
\end{aligned}
$$

In the above expressions for the Casimirs $\mathcal{F}, \mathcal{K}, g_{+}$and $g_{-}$ are arbitrary functions and we introduced the quantities

$$
D=\psi-d_{e}^{2} \nabla^{2} \psi+d_{i} v,
$$




$$
\begin{array}{r}
\omega=U+\frac{d_{i}}{c_{\beta} d^{2}} Z, \\
T_{ \pm}=\psi-d_{e}^{2} \nabla^{2} \psi+\left(d_{i}-\frac{\alpha}{c_{\beta}}\right) v \mp \frac{d_{e} \alpha^{1 / 2}}{d_{i} c_{\beta}^{1 / 2}} Z
\end{array}
$$

where $\alpha=c_{\beta} d^{2} / d_{i}, d=\sqrt{d_{i}^{2}+d_{e}^{2}}$ and $U=\nabla^{2} \varphi$ is the parallel vorticity. Note that, by making use of the variables suggested by the Casimirs, the set Eqs. (1-4) can be rewritten in the much more compact form

$$
\begin{array}{r}
\frac{\partial D}{\partial t}+[\varphi, D]=0, \\
\frac{\partial \omega}{\partial t}+[\varphi, \omega]=d_{i}^{-2}[D, \psi], \\
\frac{\partial T_{ \pm}}{\partial t}+\left[\varphi_{ \pm}, T_{ \pm}\right]=0,
\end{array}
$$

where, for convenience, we have defined

$\varphi_{ \pm}=\varphi \pm \frac{d_{\beta}}{d_{e}} \psi$.

and terms of order $d_{e}^{2} / d_{i}^{2}$ have been neglected. This form makes it evident that the fields $D, T_{+}$and $T_{-}$are Lagrangian invariants of the system and are advected by the incompressible flows associated to the stream functions $\varphi, \varphi_{+}$and $\varphi_{-}$, respectively.

This is also the form adopted for the numerical integration performed by a code based on a finite volume scheme. The averaged values of $T_{ \pm}, D$ and $\omega$ are advanced in time using an explicit third order Adams-Bashfort scheme. We choose an equilibrium configuration such that $\psi_{e q}=$ $1 / \cosh ^{2}(x / L)$, with $L=1, \phi_{e q}=v_{e q}=Z_{e q}=0$. We perturb the system adding to the equilibrium current density the following expression for $\delta J$ :

$\delta J(x, y)=J(x) \exp \left(i k_{y} y\right)$

where $k_{y}=\pi m / L_{y}, m$ is the mode wave number along $y$, and $J_{1}$ is a function localized within a width of order $d_{e}$, around the rational surface located at $x=0$. Periodic boundary conditions are imposed over the rectangular domain $(x, y):-L_{x}<x<L_{x},-L_{y}<y<L_{y}$, with $L_{x}=2 \pi$ and $L_{y}=\pi$, discretized over $1024 \times 512$ grid points.

In all the numerical investigations of collisionless reconnection we present here we restrict ourselves to the so-called large $\Delta^{\prime}$ regime, being $\Delta^{\prime}$ the standard instability parameter. This choice is motivated by the fact the this regime is relevant to the more general problem of fast reconnection, not explicitly addressed here, but firstly reported in Ottaviani and Porcelli, 1993; Aydemir, 1993; Kleva et al., 1995; Wang and Bhattacharjee, 1993.

\section{Low $\beta$ limit}

If one considers the $\beta \rightarrow 0$ limit, i.e. $c_{\beta} \rightarrow 0$, together with the $d_{i} \rightarrow \infty$ limit, then $d_{\beta} \rightarrow \rho_{s}$, the sonic Larmor radius. In this case Eq. (4) decouples from the system, Eqs. (2) and (3) give $Z=-\rho_{S} \nabla^{2} \phi$ and the all system (1-4) reduces to the standard two-field system of Schep et al. (1994). In particular, the Casimirs of the model reduce to the two generalized field $G_{ \pm}=\psi-d_{e}^{2} \nabla^{2} \psi \pm d_{e} \rho_{s} \nabla^{2} \phi$, which are also Lagrangian invariant and obey the following equations:

$\frac{\partial G_{ \pm}}{\partial t}+\left[\varphi_{ \pm}, G_{ \pm}\right]=0$

where the generalized stream functions have now become

$\varphi_{ \pm}=\varphi \pm \rho_{s} / d_{e} \psi$

This low $\beta$ limit has been extensively studied in the literature Grasso et al., 2001; Cafaro et al., 1998; Del Sarto et al., 2003, 2005, 2006 and we recall here its main features, recovering the aforementioned results by solving the full four-field system of Eqs. (1-4).

When the electron temperature effects are negligible and $d_{\beta}=\rho_{s} \rightarrow 0$, the system of Eq. (19) degenerate, since $G_{+}$coincides with $G_{-}$. An expansion to first order in the $\rho_{s}$ parameter is necessary in order to recover the equations for the canonical momentum, $F=\psi+d_{e}^{2} J$, and the vorticity, $U$ (Cafaro et al., 1998). In this limit $F$ becomes the only Lagrangian invariant of the model. $F$ is advected by the velocity field $v=\nabla \varphi \times \mathbf{e}_{z}$. Its field lines are pushed towards each other and concentrate in a narrow region of the order of $d_{e}$. Consequently, the current density and vorticity layers tend to align along the neutral line of the initial equilibrium configuration. In this situation strong velocity shears develop and, when the reconnection process has reached saturation, these layers undergo a secondary instability of the Kelvin-Helmholtz type (Del Sarto et al., 2003). This coupling of the reconnection instability with the hydrodynamical one lead to the development of turbulence inside the magnetic island. Two jets generate in the current density and vorticity layers in correspondence of the $X$-point and move towards the $O$-point, where they collide and change direction, moving to the edge of the island, where they destabilize. Here we solve the four-field model equations assuming $c_{\beta}=0.001, d_{e}=0.24$, and $d_{\beta}=0.0024$. A sequence of the dynamical steps described above is illustrated in Fig. 1, where the contour plots of the vorticity field (first row) and of the current density (second row) are shown at $t=40,45,50 \tau_{A}$.

A completely different scenario shows up when the electron temperature effects are retained, and $d_{\beta}=\rho_{s} \neq 0$. In this case, the fields $G_{ \pm}$, advected by the velocity fields $v_{ \pm}=\nabla \varphi_{ \pm} \times \mathbf{e}_{z}$, rotate in opposite directions (Cafaro et al., 1998), while conserving their topology, resembling a baker transformation. In this way, they undergo a phase mixing process (Grasso et al., 2001), in the advanced nonlinear phase. The current density and the vorticity, which correspond to the sum and the difference of $G_{ \pm}$, respectively, reflect the rotation assuming a cross-structure shape aligned with the branches of the separatrix of the magnetic island and 

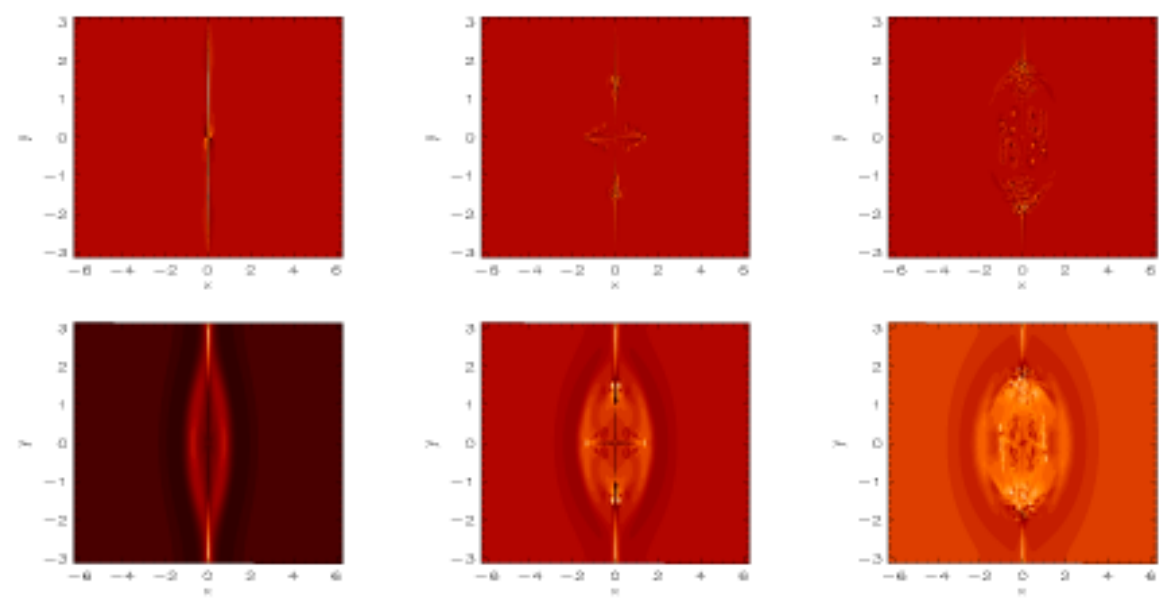

Fig. 1. Contour plots of the vorticity (top row) and of the current density (bottom row) illustrating the turbulent regime occurring in the low $\beta$ limit of the four-field model for a case with $c_{\beta}=0.001, d_{e}=0.24$, and $d_{\beta}=0.0024$. The three columns represent $40,45,50 \tau_{A}$, respectively.
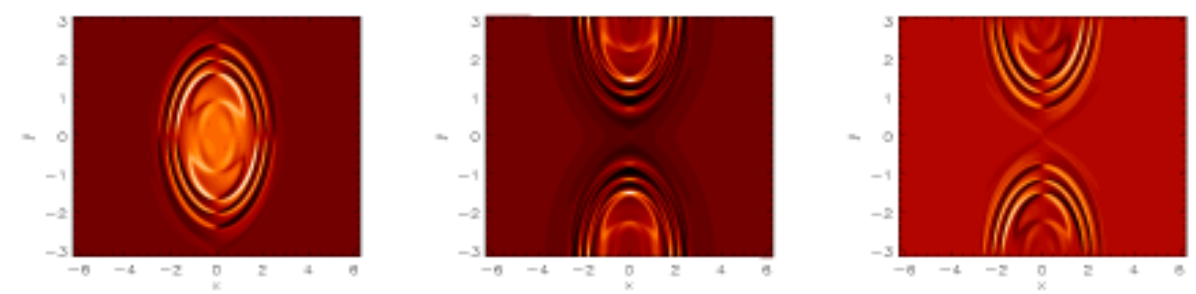

Fig. 2. Contour plots of the $G_{+}$field (left frame), current density (middle frame) and vorticity (right frame) illustrating at $t=70 \tau_{A}$, the laminar regime occurring in the low $\beta$ limit of the four-field model for a case with $c_{\beta}=0.001, d_{e}=0.24$, and $d_{\beta}=0.24$.

reflect the phase mixing process exhibiting a laminar structure that becomes more and more refined as the time goes on. A typical example of this behavior is given in Fig. 2 where a snapshot of $G_{+}, J$ and $U$ is given in the saturation phase, at $t=70 \tau_{A}$, for a case with $c_{\beta}=0.001, d_{e}=0.24$, and $d_{\beta}=0.24$. We can clearly appreciate here the filamented structure that characterizes this regime.

\subsection{3-D extension}

The occurrence of secondary instability on the top of the reconnection one in the $\rho_{s} \rightarrow 0$ limit has already been observed also in a three dimensional context (Grasso et al., 2007). The three-dimensional extension of the two field-model in Eq. (19) reads as Borgogno et al. (2005):

$\frac{\partial G_{ \pm}}{\partial t}+\left[\varphi_{ \pm}, G_{ \pm}\right]=\frac{\partial\left[\varphi_{ \pm} \mp\left(\varrho_{s} / d_{e}\right) G_{ \pm}\right]}{\partial z}$.

3-D effects introduce magnetic field lines chaoticity (Borgogno et al., 2008). Nevertheless, layers of vorticity and current density persist even in such a chaotic setting. In Grasso et al. (2007), these layers have been shown to undergo secondary instability of the hydrodynamic type, when the growth rate of the reconnection process is around its higher value in the cold electron case. Here we present new simulations that support our findings. In particular, we have performed a high resolution run, which allows us to highlight the intense shear layers formation and their following destabilization. This 3-D simulation has been carried out adopting the code described in Grasso et al. (2007), that solves Eq. (20) starting from a static equilibrium configuration whit an Harris-type magnetic field such that $B_{e q}=\tanh (x)$. The simulation has been carried out in a slab geometry defined by $-L_{x}<x<L_{x},-L_{y}<y<L_{y}$, and $-L_{z}<z<L_{z}$, with $801 \times 512 \times 512$ grid points. Dirichlet boundary conditions have been imposed along the $x$ direction, while periodicity has been used along $y$ and $z$, for all the perturbed fields.

In order to consider the 3-D effects on the reconnection, we destabilize the initial equilibrium by the following linearly unstable, double helicity current perturbation

$$
\begin{aligned}
\delta J(x, y, z) & =\delta \hat{J}_{1}(x) \exp \left(i k_{y 1} y+i k_{z 1} z\right) \\
& +\delta \hat{J}_{2}(x) \exp \left(i k_{y 2} y+i k_{z 2} z\right)
\end{aligned}
$$

where $k_{y}=\pi m / L_{y}, k_{z}=\pi n / L_{z}$ and $(m, n)$ are the mode wave numbers along $y$ and $z$, while $\delta \hat{J_{1,2}}(x)$ are functions 

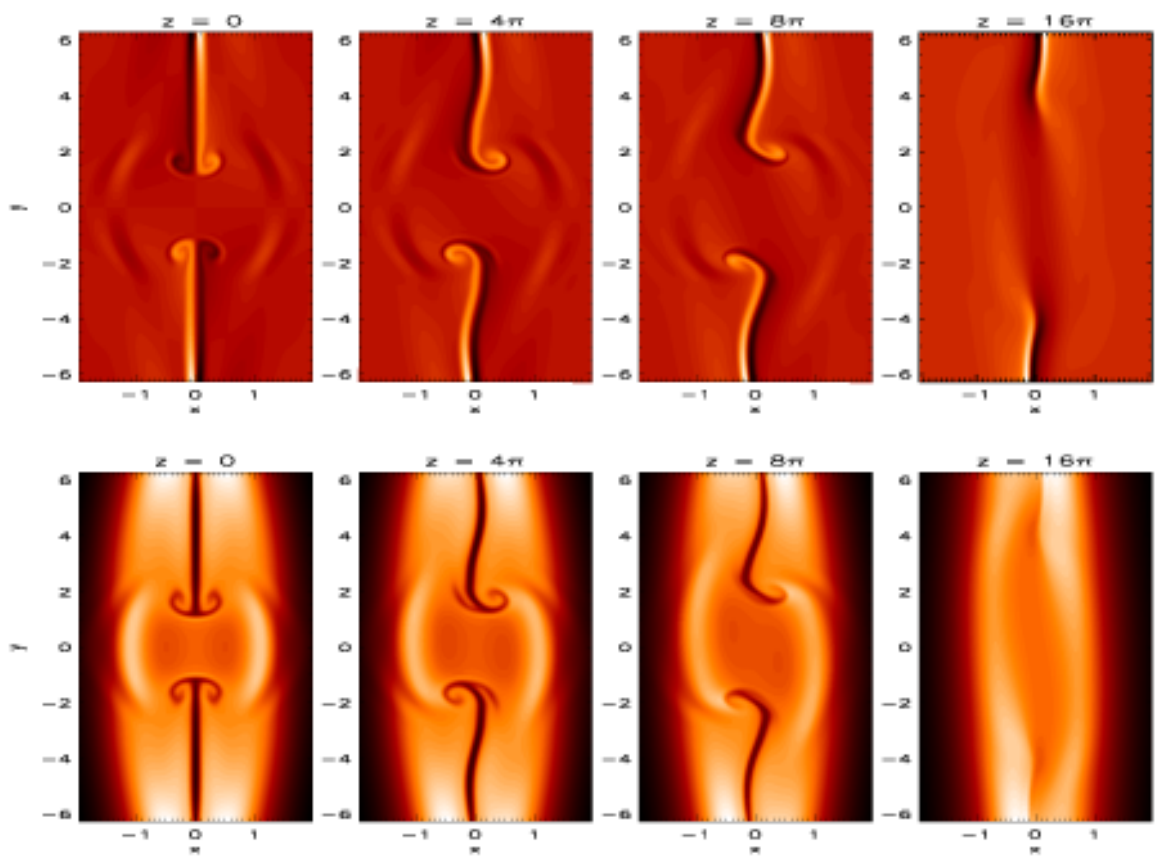

Fig. 3. Contour plots of the vorticity (top row) and of the current density (bottom row) on different $z=$ constant planes, at $t=210 \tau_{A}$, illustrating the onset of the Kelvin-Helmholtz instability on a three-dimensional reconnection process in the low $\beta$ limit when $\rho_{s} \rightarrow 0$.

localized within a layer of order $d_{e}$, around the resonant surfaces corresponding to the two helicities. In the results reported here we choose $d_{e}=0.3, \rho_{s}=0.03, L_{x}=11.36$, $L_{y}=4 \pi, L_{z}=64 \pi$ and $k_{y 1}=k_{y 2}=1 / 2, k_{z 1}=-k_{z 2}=1 / 32$.

In the 3-D context the nonlinear interaction between the different helicities depends on the $z$ coordinate, being stronger when the magnetic islands, corresponding to the initial perturbations, face each other (Grasso et al., 2007). Consequently, the developing of the Kelvin-Helmholtz instability starts at different time on the different $z$ constant planes. Figures 3, 4 illustrate a zoom on the $(x, y)$ plane of the shaded contours of the current density (top row) and vorticity (bottom row) on the four sections $z=0, z=4 \pi, z=8 \pi$, $z=16 \pi$ at the nonlinear times $t=210 \tau_{A}$ and $t=220 \tau_{A}$, respectively. At $t=210 \tau_{A}$ the interaction between the two different helicity modes initially imposed leads to the formation of a pair of bar-shaped patterns whose width is smaller than $d_{e}$. The presence of the characteristic vortex-rings, at this time clearly visible on the sections $z=0, z=4 \pi$ and $z=8 \pi$, shows the fluid behavior of the dynamics inside the sheets. At $t=220 \tau_{A}$ (Fig. 4) the current density and vorticity fields start to be affected by the Kelvin-Helmholtz instability an all the $z$ constant planes. The patterns of the layers appear rather disrupted and the small scale characteristic vortexes has already developed.

As a comparison we show in Fig. 5 the contour plots of the vorticity and the current density for a case with $\rho_{s} \neq 0$. We point out that the laminar structure, typical of the evolution of the $G_{ \pm}$fields, is clearly visible on all the different planes.

\section{High $\beta$ limit}

Here we explore the nonlinear dynamics of the system of Eqs. (1-4) when finite $\beta$ effects are retained. Preliminary results presented in Tassi et al. (2007, 2008a) have shown the appearance of a new regime where the occurrence of secondary hydrodynamical instability as a byproduct of the reconnection one presents new features. In particular, the typical coupling between the behavior of the current density and vorticity layers is now broken, allowing for the coexistence of laminar and turbulent regimes. In particular, we observe that, increasing the value of $c_{\beta}$, while the current density retain the laminar structure observed in the low $\beta$ limit, the vorticity eventually develops a hydrodynamical instability ending up, for sufficiently high $c_{\beta}$ values, in a totally turbulent regime. An example of this different behavior is shown in Fig. 6, where the contour plots of the vorticity and of the current density are given at $t=65 \tau_{A}$, a time well into the saturated phase of the reconnection process, for a simulation with $c_{\beta}=0.4, d_{e}=0.24$, and $d_{\beta}=0.96$. It is interesting to note that in the vorticity there is a sort of competition between the laminar and turbulent regimes, when the secondary instability develops. The turbulence can fully develop and destroy the laminar structure spreading all inside the island depending on the value of $c_{\beta}$. This double aspect of the vorticity can be observed in Fig. 7, where a sequence in time is plotted for a simulation with $c_{\beta}=0.1, d_{e}=0.24$, and $d_{\beta}=0.24$. More precisely, snapshots at $t=75,90,100 \tau_{A}$ are shown. 

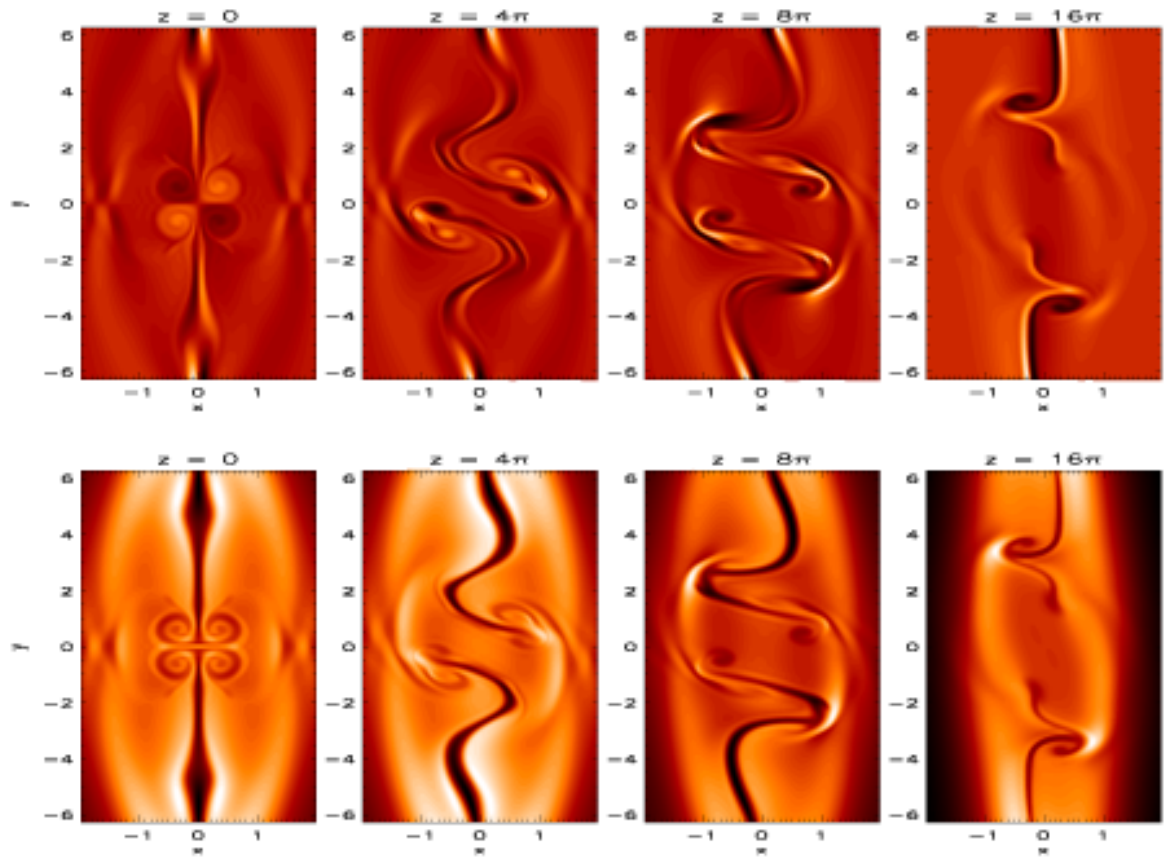

Fig. 4. Contour plots of the vorticity (top row) and of the current density (bottom row) on different $z=$ constant planes, at $t=220 \tau_{A}$, illustrating the developing of the turbulent regime in a three-dimensional reconnection process in the low $\beta$ limit when $\rho_{S} \rightarrow 0$.
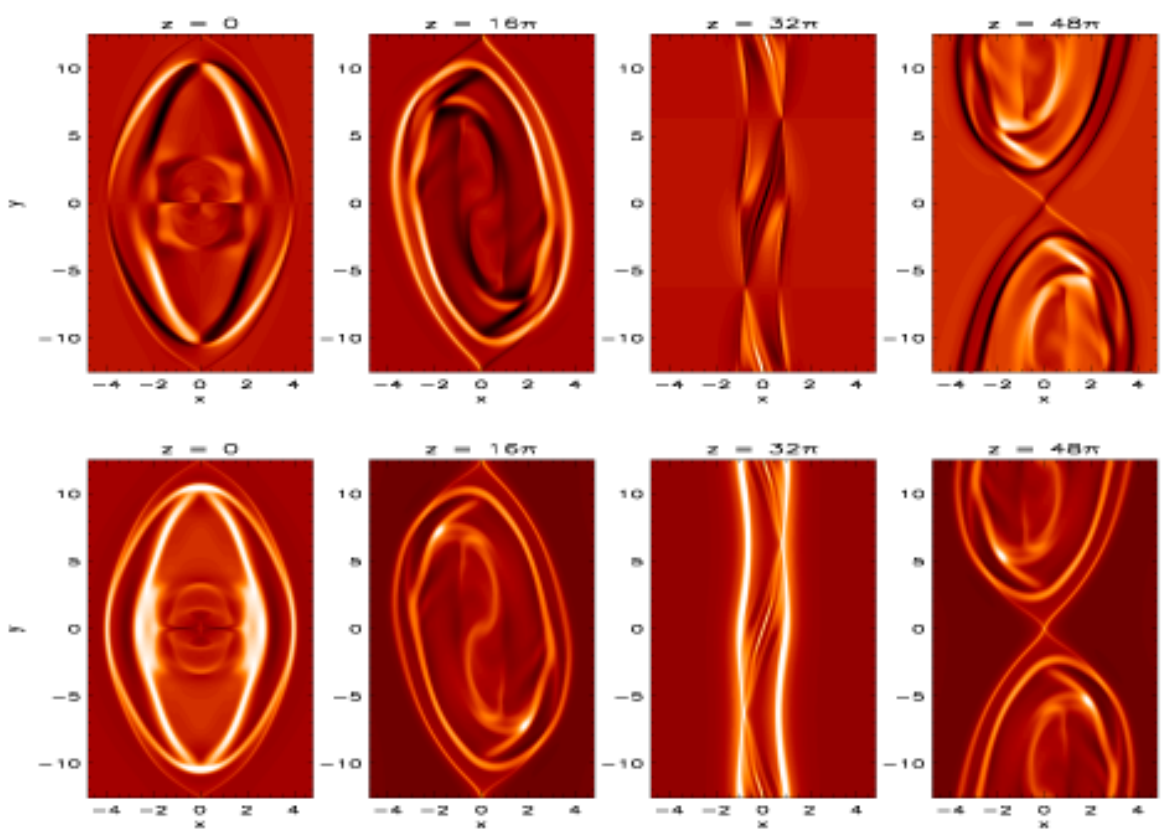

Fig. 5. Contour plots of the vorticity (top row) and of the current density (bottom row) on different $z=$ constant planes, at $t=200 \tau_{A}$, illustrating the developing of the laminar structures in a three-dimensional reconnection process in the low $\beta$ limit when $\rho_{s} \neq 0$.

This coexistence of opposite regimes can be explained in terms of the richer Lagrangian structure of the four-field model. Indeed, we observe that, in this intermediate regime, the two invariants $T_{ \pm}$, advected by $\varphi_{ \pm}$, undergo a phase mix- ing process in the saturation phase, while $D$ and $\omega$ on top of the reconnection process develop a Kelvin-Helmholtz instability. Figure 8 shows the contour plots of $T_{ \pm}$in the left and right frame in the top row, and of $D$ and $\omega$ in the left and right 

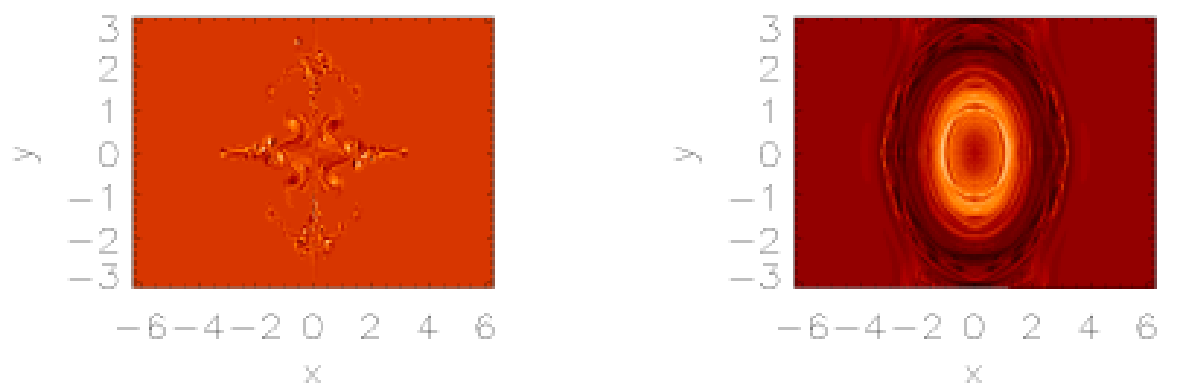

Fig. 6. Contour plots of the vorticity (left frame) and of the current density (right frame) at $t=65 \tau_{A}$ for a case in the higher $\beta$ limit, with $c_{\beta}=0.4, d_{e}=0.24$, and $d_{\beta}=0.96$.
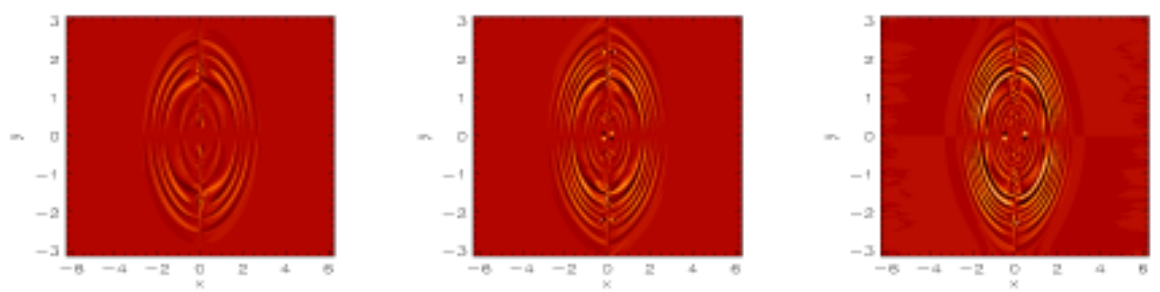

Fig. 7. Sequence in time $\left(t=75,90,100 \tau_{A}\right)$ of the contour plot of the vorticity for a simulation with $c_{\beta}=0.1, d_{e}=0.24$, and $d_{\beta}=0.24$.
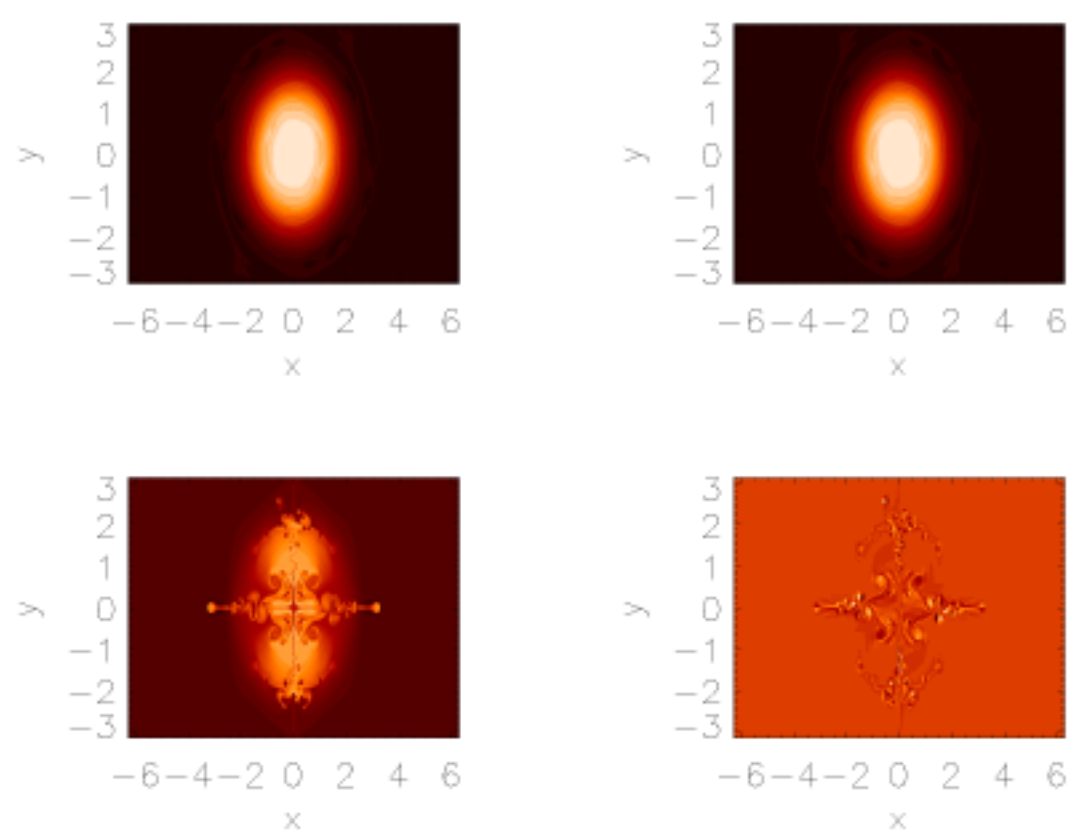

Fig. 8. Contour plots of $T_{ \pm}$in the left and right frame in the top row, and of $D$ and $\omega$ in the left and right frame in the bottom row, for the same case shown in Fig. 6.

frame in the bottom row, for the same case shown in Fig. 6 . This twofold evolution of the invariants of the model reflects in the behavior of the current density and vorticity layers. While the current density evolves according to the laminar structure related to the mixing of the Lagrangian invariants $T_{ \pm}$, the vorticity layers initially show the same structure but at later times are dominated by the evolution of the $\omega$ field and undergo secondary instabilities taking a turbulent behavior. If we consider the relationships between the invariant fields and $U$ and $J$ we find that:

$$
J=\frac{T_{+}+T_{-}}{2 d_{e}^{2}}-\frac{\psi}{d_{e}^{2}}+\frac{D}{d_{i}^{2}}, \quad U=\frac{T_{+}-T_{-}}{2 d_{e} d_{\beta}}+\omega .
$$


From these expressions it is clear that the behaviors of $J$ and $U$ differ due to the presence of $\omega$ in the latter. The field $\omega$ is responsible for the formation of two vertical jets that propagate towards each other and that, upon colliding, form vortex pairs and eventually undergo a Kelvin-Helmholtz type of instability. We recall that the $\omega$ field is related to the $Z$ field, which is physically responsible for compression of the magnetic field along the $z$ direction.

\section{Conclusions}

In conclusion we have investigated the coupling between magnetic reconnection and Kelvin-Helmholtz instability in the framework of the two-dimensional four-field model for collisionless regimes. In particular, we have analyzed the different regimes that occur depending on the value of the $\beta$ parameter. We find that while, in the low $\beta$ limit, the hydrodynamical instability develops on the top of the reconnection process only in the cold electron regime, increasing the value of $\beta$ opens different scenarios.

In particular, when $\beta \rightarrow 0$ two distinct regimes can be identified depending on the value of the electron temperature, which enters the equations through the ion sound Larmor radius, $\rho_{s}$. Mainly, when electron temperature effects are taken into account for, the advanced nonlinear phase of the reconnection process is characterized by a laminar regime in the vorticity and current density dynamical evolution. On the contrary, when these effects are neglected and $\rho_{s} \rightarrow 0$, the same fields, after the occurrence of a Kelvin-Helmholtz like instability, develop a turbulent regime. The main feature we intend to highlight, in this low $\beta$ limit, is that the dynamical evolution of the current density and vorticity layers is coupled, i.e. they evolve according to the same laminar or turbulent regime. This coupling is explained in terms of the Lagrangian invariants of the model. These results, already known in the literature in the framework of the twofield model Grasso et al. (2001); Del Sarto et al. (2003), have been here derived in the more general context of the fourfield model.

This coupling between the behavior of the current density and the vorticity layers persist also when three-dimensional effects are considered (Grasso et al., 2007). Here, we illustrate these aspects showing new high resolution 3-D simulations solving directly the two-field model equations in the $\rho_{s} \rightarrow 0$ limit.

When considering finite values of the $\beta$ parameter, allowing for compression of the magnetic field along the ignorable direction, the dynamical evolution of the current density and vorticity sheets decouple from each other. While the current density maintains a laminar structure, the vorticity undergoes secondary instabilities ending up in a turbulent regime. The richer Lagrangian structure of the model gives account for this different behavior. Indeed, the vorticity field can be decomposed into a first component, related to the Lagrangian invariants $T_{ \pm}$, that is responsible for the filamentation on small scales, and to a second component, corresponding to the field $\omega$, that accounts for the jet formation and dynamics.

Acknowledgements. This work was partly supported by the Euratom Communities under the e contract of Association between EURATOM/ENEA. The views and opinions expressed herein do not necessarily reflect those of the European Commission. This work was also partly supported by a grant PRIN-2006.

Edited by: T. Passot

Reviewed by: two anonymous referees

\section{References}

Aydemir, A.: Nonlinear studies of $m=1$ modes in high-temperature plasmas, Phys. Fluids B, 4, 3469, doi:10.1063/1.860355, 1992.

Borgogno, D., Grasso, D., Califano, F., Farina, F., Pegoraro, F., and Porcelli, F.: Aspects of three-dimensional magnetic reconnection, Phys. Plasmas., 12, 032309, doi:10.1063/1.1857912, 2005.

Borgogno, D., Grasso, D., Pegoraro, F., and Schep, T. J.: Stable and unstable invariant manifolds in a partially chaotic magnetic configuration generated by nonlinear reconnection, Phys. Plasmas, 15, 102308, doi:10.1063/1.2999539, 2008.

Cafaro, E., Grasso, D., Pegoraro, F., Porcelli, F., and Saluzzi, A.: Invariants and Geometric Structures in Nonlinear Hamiltonian Magnetic Reconnection, Phys. Rev. Lett, 80(20), 4430-4433, doi:10.1103/PhysRevLett.80.4430, 1998.

Del Sarto, D., Califano, F., and Pegoraro, F.: Secondary Instabilities and Vortex Formation in Collisionless-Fluid Magnetic Reconnection, Phys. Rev. Lett, 91(23), 235001-1, doi:10.1103/PhysRevLett.91.235001, 2003.

Del Sarto, D., Califano, F., and Pegoraro, F.: Current layer cascade in collisionless electron-magnetohydrodynamic reconnection and electron compressibility effects, Phys. Plasmas, 12, 012317, doi:10.1063/1.1829064, 2005.

Del Sarto, D., Califano, F., and Pegoraro, F.: Electron Parallel Compressibility in the nonlinear Development of Two-Dimensional Collisionless Magnetohydrodynamic Reconnection, Modern Phys. Lett. B, 20, 931-961, doi:10.1142/S0217984906011621, 2006.

Fitzpatrick, R. and Porcelli, F.: Collisionless magnetic reconnection with arbitrary guide-field, Phys. Plasmas, 11, 4713, 2004; Phys. Plasmas, 14, 049902, erratum, 2007.

Grasso, D., Califano, F., Pegoraro, F., and Porcelli, F.: Phase Mixing and Island Saturation in Hamiltonian Reconnection, Phys. Rev. Lett., 86, 5051, doi:10.1103/PhysRevLett.86.5051, 2001.

Grasso, D., Borgogno, D., and Pegoraro, F.: Secondary instabilities in two- and three-dimensional magnetic reconnection in fusion relevant plasmas, Phys. Plasmas,14, 055703-1, doi:10.1063/1.2436483, 2007.

Kleva, R. G., Drake, J. F., and Waelbroeck, F. L.: Fast reconnection in high temperature plasmas, Phys. Plasmas, 2(23), 23-34, doi:10.1063/1.871095, 1995.

Morrison, P. J.: Hamiltonian description of the ideal fluid, Rev. Mod. Phys., 70, 467-521, doi:0.1103/RevModPhys.70.467, 1998. 
Ottaviani, M. and Porcelli, F.: Nonlinear collisionless magnetic reconnection, Phys. Rev. Lett., 71(23), 3802, doi:10.1103/PhysRevLett.71.3802, 1993.

Schep, T. J., Pegoraro, F., and Kuvshinov, B. N.: Generalized twofluid theory of nonlinear magnetic structures, Phys. Plasmas, 9, 2843, doi:10.1063/1.870523, 1994.

Tassi, E., Morrison, P. J., and Grasso, D.: Hamiltonian Structure of a Collisionless Reconnection Model Valid for a High and Low $\beta$ Plasmas, in: Collective Phenomena in Macroscopic Systems, World Scientific, Singapore, p. 197, 2007.
Tassi, E., Grasso, D., and Pegoraro, F.: Nonlinear processes in Hamiltonian reconnection, Commun. Nonlinear Sci. Numer. Simulat., in press, 2008a.

Tassi, E., Morrison, P. J., Waelbroeck, F. L., and Grasso, D.: Hamiltonian formulation and analysis of a collisionless fluid reconnection model, Plasma Phys. Contr. F., 50, 085014, doi:10.1088/0741-3335/50/8/085014, 2008b.

Wang, X. and Bhattacharjee, A.: Nonlinear dynamics of the $m=1$ instability and fast sawtooth collapse in high-temperature plasmas, Phys. Rev. Lett., 70, 1627, doi:10.1103/PhysRevLett.70.1627, 1993. 